\title{
Intelligentes Rekonfigurationsmanagement selbstorganisierter Produktionssysteme in der diskreten Fertigung
}

\author{
T. Müller, M.Sc.; Dr.-Ing. N. Jazdi; Univ.-Prof. Dr.-Ing. M. Weyrich, \\ Universität Stuttgart, Institut für Automatisierungstechnik und \\ Softwaresysteme, Stuttgart
}

\section{Kurzfassung}

Die Häufigkeit von Änderungen der Produktionsanforderungen nimmt aufgrund wirtschaftlicher Volatilität, kürzerer Innovationszyklen und Produktlebenszyklen kontinuierlich zu. Daher ist eine Vorhersage aller möglichen Ziele eines Produktionssystems zur Entwurfszeit unmöglich und es ergibt sich erhöhter Rekonfigurationsbedarf zur Betriebszeit. Derzeit weist die Rekonfiguration von Produktionssystemen jedoch einige Schwachstellen auf, die in diesem Beitrag aufgezeigt werden. Außerdem wird die Zukunft der industriellen Automatisierung von Cyber-Physischen Produktionssystemen dominiert werden, welche vielversprechende Potentiale bieten. Folglich werden die Cyber-Physischen Produktionssysteme und einige ihrer Potentiale im Hinblick auf Rekonfiguration diskutiert. Um diese theoretischen Potentiale tatsächlich nutzen zu können, sind allerdings entsprechende Konzepte erforderlich, weshalb dieser Forschungsbeitrag ein grundlegendes Konzept für ein selbstorganisiertes Rekonfigurationsmanagement präsentiert.

\section{Einleitung}

Die Zukunft der industriellen Automatisierung wird, wie auch die Literatur oftmals zeigt [1-3], vom Konzept der Cyber-Physischen Systeme (CPS) geprägt sein. Kernaspekte solcher CPS sind (basierend auf [4]), neben ihren physischen Komponenten, ihre Konnektivität und ihre Fähigkeiten zur Informationsverarbeitung. Diese ermöglichen es CPS, einen Grad an Intelligenz inne zu haben, der in seinen konkreten Ausprägungen stark variieren kann. Produktionssysteme, die aus CPS bestehen, werden auch als Cyber-Physische Produktionssysteme (CPPS) bezeichnet [3]. Einerseits bieten diese Systeme erhebliche theoretische Potentiale für die Automatisierungstechnik von morgen, von denen einige in diesem Artikel hervorgehoben werden. Andererseits ergeben sich aber auch neue Herausforderungen, wie z.B. die Beherrschung der zunehmenden Komplexität, die Ermöglichung einer dynamischen Vernetzung oder das Finden geeigneter Konzepte zur Nutzung dieser theoretischen Potentiale. 
Neben dem sich abzeichnenden Trend der CPPS [5] in der Industrieautomatisierung erfordern die zunehmende Volatilität in der globalen und lokalen Wirtschaft, die Verkürzung der Innovations- und Produktlebenszyklen sowie eine enorm steigende Variantenvielfalt Produktionssysteme, die diesen Anforderungsänderungen gerecht werden [6]. Diese sich ändernden Anforderungen führen dazu, dass die konkreten Zielsetzungen für Produktionssysteme in der Phase des Systementwurfs immer unvorhersehbarer werden. Daher werden Änderungen am System während des Betriebs zur Regel statt zur Ausnahme $[7,8]$. Insbesondere wenn diese Anforderungsänderungen den Flexibilitätskorridor (d.h. die aktuelle Funktionalität) des im Voraus geplanten Produktionssystems überschreiten, wird eine Rekonfiguration notwendig. Der aufgezeigte steigende Rekonfigurationsbedarf während der Betriebszeit wird, neben vielen anderen, auch in [9-11] angemerkt.

\section{Grundlagen, Potenziale und Herausforderungen}

\subsection{Rekonfiguration und ihre derzeitigen Schwächen}

In diesem Beitrag wird die folgende Definition von Rekonfiguration verwendet: „Eine Rekonfiguration stellt die technische Sicht des Prozesses der Veränderung eines bereits entwickelten und operativ eingesetzten Systems dar, um es an neue Anforderungen anzupassen, Funktionalität zu erweitern, Fehler zu beseitigen oder die Qualitätseigenschaften zu verbessern." [12]

Diese Thematik umfasst jedoch nicht nur die Durchführung von Rekonfigurationsmaßnahmen. Mindestens ebenso wichtig sind die Ermittlung des Rekonfigurationsbedarfs während des Betriebs und die zuvor erforderliche Rekonfigurationsplanung. Daher wird der Begriff des Rekonfigurationsmanagements einführt, der die Ermittlung des Rekonfigurationsbedarfs, die Rekonfigurationsplanung und die Durchführung von Rekonfigurationsmaßnahmen (optional) umfasst.

Obwohl die wirtschaftliche und ökologische Notwendigkeit der Rekonfiguration von Produktionssystemen unumstritten ist, wird sie bisher kaum realisiert [10, 13]. 
Dies ist darauf zurückzuführen, dass die Anpassung von Produktionssystemen derzeit einige Schwachstellen aufweist [14, 15]:

- zeitaufwändig, da manuell und individuell ausgelöst, geplant und durchgeführt.

- fehleranfällig, weil sie nicht systematisch oder methodisch unterstützt wird.

- keine Garantie für eine optimumnahe Lösung, da sich auf begrenztes menschliches Wissen gestützt wird und daher nur eine Teilmenge des Lösungsraums für alternative Konfigurationen berücksichtigt wird.

- Bewertung und Auswahl folgen keinen objektiven Kriterien, sondern basieren auf menschlicher Erfahrung.

Diese Defizite sollen mit dem später vorgestellten Konzept für das Rekonfigurationsmanagement adressiert werden. Darüber hinaus hat auch [16] die Notwendigkeit von Methoden erkannt, welche die Wandlungsfähigkeit in Produktionssysteme bringen. Ein wichtiger Aspekt bei der Entwicklung wandlungsfähiger Produktionssysteme ist, dass Produktionsressourcen schnell (re-) konfiguriert und eingesetzt werden können, um sich ändernden Anforderungen gerecht zu werden [16].

Des Weiteren hängen der Erfolg und die Akzeptanz rekonfigurierbarer Produktionssysteme nach [13] und [17] in erster Linie vom Aufwand für die Rekonfiguration des Systems und dem daraus resultierenden Nutzen ab. Um den Aufwand zu reduzieren, kann grundsätzlich eine erhöhte Rekonfigurierbarkeit des Systems angestrebt werden oder es wird versucht die notwendigen manuellen Tätigkeiten beim Rekonfigurationsmanagement zu reduzieren Insbesondere letzteres wird im Rahmen dieses Artikels durch die Vorstellung eines geeigneten Konzepts für das Rekonfigurationsmanagement adressiert.

Um dieses Ziel zu erreichen, müssen zunächst Kenntnisse über die aktuelle Konfiguration des Produktionssystems (z.B. in Form von Modellen) als notwendige Voraussetzung vorhanden sein. Sowohl die Automatisierung des Rekonfigurationsmanagements als auch die Identifizierung der aktuellen Konfiguration des Produktionssystems können durch die Potentiale, die CPPS theoretisch bieten, angegangen werden.

\subsection{CPPS und ihre Potentiale}

CPS verbinden physische Objekte und Prozesse mit virtuellen informationsverarbeitenden Objekten und Prozessen [18]. Im Gegensatz zu traditionellen eingebetteten Systemen, die als eigenständige Geräte konzipiert sind, liegt der Fokus bei CPS auf der Vernetzung mehrerer Geräte [1].

CPPS haben mehrere Wurzeln innerhalb der Produktionsdomäne, einige davon sind: Intelligente Produktionssysteme (IMS), rekonfigurierbare Produktionssysteme (RMS), biologische Produktionssysteme (BMS) und digitale Fabriken (DF) [19]. Folglich soll das 
Konzept der CPPS nicht im Widerspruch zu diesen Entwicklungen stehen, sondern diese, zumindest teilweise, zusammenführen und weiter verbessern.

Durch ihre hohe Vernetzung stellen CPPS die „Vision von adaptiven, sich selbst konfigurierenden und teilweise selbstorganisierenden, flexiblen Produktionsanlagen" [20] dar und können zu geringeren Rüstzeiten und einem optimiertem Energie- und Ressourceneinsatz [20] führen.

Um jedoch die Potentiale von CPPS, wie beispielsweise ein selbstorganisiertes Rekonfigurationsmanagement erschließen zu können, müssen zunächst Voraussetzungen wie die Existenz von semantischen Beschreibungen des CPPS und seiner Komponenten erfüllt sein. In diesem Zusammenhang kam [21] zu dem Schluss, dass die Ausschöpfung des vollen Potentials von CPS semantische Modelle erfordert. Aufbauend auf diesen semantischen Informationen, können entsprechende Konzepte entwickelt und in die Anwendung überführt werden.

Darüber hinaus besteht ein Bedarf an Konzepten, die bestehende Algorithmen aus Domänen wie der Künstlichen Intelligenz oder der mathematischen Optimierung, gegebenenfalls in angepasster Form, übernehmen [20]. Beispiele hierfür sind [22] für die Bilderkennung und [23] für die prädiktive Qualitätskontrolle, die beide in industriellen Automatisierungssystemen Anwendung finden.

„In CPPS werden Daten, Dienste und Funktionen dort gehalten, abgerufen und ausgeführt, wo es den größten Nutzen in Bezug auf flexibles und effizientes Design, Engineering, Entwicklung und Produktion bringt" [24]. Daraus wird in [24] abgeleitet, dass sich die klassische Automatisierungspyramide schrittweise auflösen und durch vernetzte, dezentral organisierte Systeme ersetzt wird.

Ein Cyber-Physisches Produktionssystem besteht aus mehreren Cyber-Physischen Produktionsmodulen (CPPM), welche wiederum neben ihren physischen Komponenten aus ihrer Modulsteuerung und ihrem Cyber-Teil mit seiner Intelligenz und seinen Modellen bestehen. Das CPPS umfasst somit die darin enthaltenen CPPM und stellt so das komplette Produktionssystem dar. Darüber hinaus enthält das CPPS seine eigenen Cyber-Komponenten und physischen Komponenten, die für die Organisation und den Betrieb des Gesamtsystems notwendig sind. Die sich daraus ergebende, neuartige CPPS-Struktur ist in Bild 1 dargestellt. Diese wird durch VDI-Definitionen und [1] gestützt und ist aufgrund ihrer Modularität geeignet, dem steigenden Bedarf an Rekonfiguration Rechnung zu tragen. 


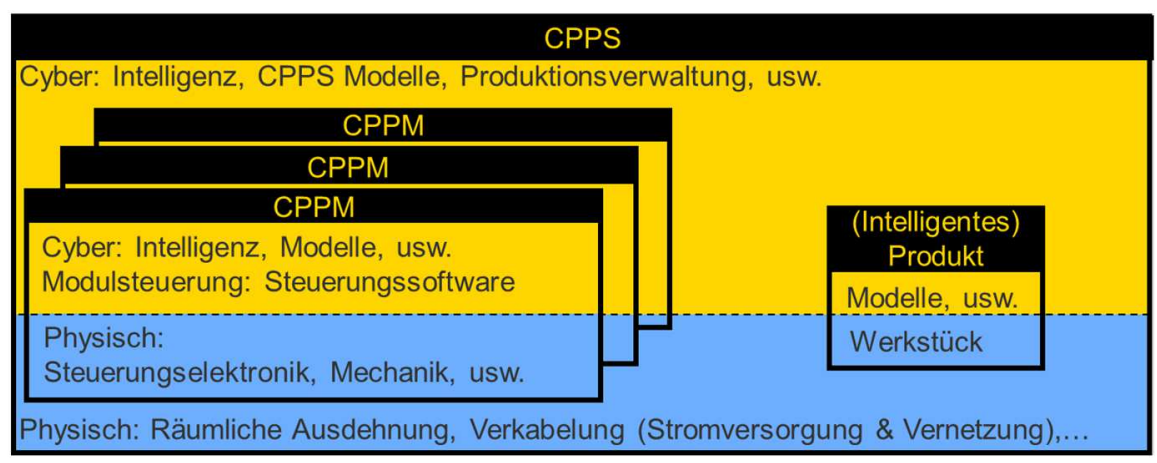

Software Hardware

Bild 1: Struktur von CPPS und deren Bestandteile

In den vergangenen Jahren wurden mehrere Industrie 4.0-Modellfabriken und Testumgebungen geschaffen. Ein Überblick der deutschen Aktivitäten ist in [25] aufgeführt. Des Weiteren stellt der digitale Zwilling, als Konzept zur virtuellen Abbildung von Gegenständen der physischen Welt in die Informationswelt [26], einen großen Fortschritt bezüglich Modellierungs-, Simulations- und Optimierungstechnologien, im Rahmen von CPS, dar [27]. Ein erster Schritt zur Nutzung eines digitalen Zwillings im Kontext der Rekonfiguration von CPPS wurde in [28] beschrieben.

CPPS bieten also hohe Potentiale für das oben beschriebene Rekonfigurationsmanagement, insbesondere da sie über Modelle verfügen und Intelligenz bieten können, um dies auf selbstorganisierte Weise zu erreichen. Es stellt sich aber auch die Frage, wie CPPS um die Fähigkeit eines selbstorganisierten Rekonfigurationsmanagements bereichert werden können?

\section{Konzept für das selbstorganisierte Rekonfigurationsmanagement}

Ein grundlegendes Konzept zur Beantwortung dieser Forschungsfrage wird in diesem Kapitel vorgestellt. Hierzu wird in Abschnitt 3.1 die Methodik für den Ablauf des Rekonfigurationsmanagement vorgestellt, gefolgt von der Verortung der Intelligenz in Abschnitt 3.2.

\subsection{Methodik für das Rekonfigurationsmanagement}

Bei der Entwicklung dieser Methodik wurde der Ansatz des Design Thinkings angewandt. Insbesondere bedeutet dies, dass Experten aus verschiedenen Fachdisziplinen dazu beigetragen haben eine möglichst umfassende Methodik zu definieren. Diese beinhaltet 4 Schritte, welche in den folgenden Abschnitten erläutert werden.

(1) Ermittlung des Rekonfigurationsbedarfs: Um ein selbstorganisiertes Rekonfigurationsmanagement zu ermöglichen, muss das CPPS zunächst einen bestehenden Rekonfigurationsbedarf während des Betriebs erkennen können. 
Bild 2 zeigt eine vereinfachte Beschreibung des Verfahrens, mit dem dieses Problem gelöst werden soll. Es gibt zwei verschiedene Trigger, die eine Überprüfung auf einen bestehenden Rekonfigurationsbedarf auslösen können. Zum einen können sich die Anforderungen aufgrund neu erteilter Produktionsaufträge ändern. Zum anderen lösen Komponentenausfälle innerhalb des Produktionssystems eine Überprüfung aus.

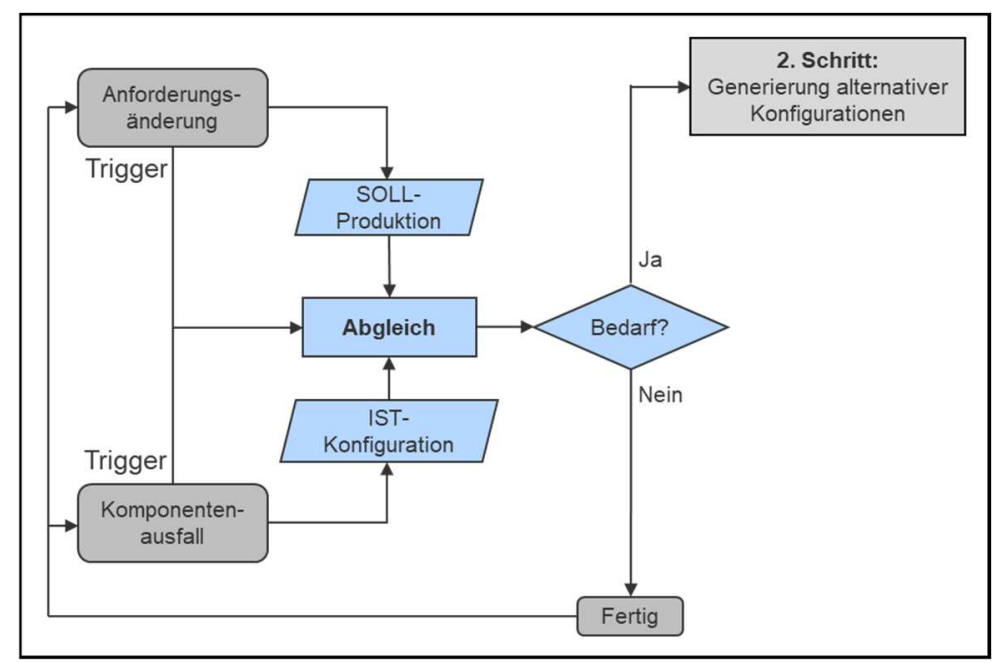

Bild 2: Ermittlung des Rekonfigurationsbedarfs (vereinfachte Darstellung)

Die SOLL-Produktion besteht aus der Beschreibung der Produkte basierend auf den erforderlichen einzelnen Produktionsprozessen sowie deren Reihenfolge. Darüber hinaus beinhaltet sie die Gewichtung der Kriterien, die für die spätere Bewertung der Konfigurationen benötigt wird. Die IST-Konfiguration enthält eine Beschreibung aller aktuell eingesetzten Produktionsressourcen (CPPM) des CPPS. Dazu gehören ihre Fähigkeiten zur Durchführung von Produktionsprozessen, einschließlich ihrer einstellbaren Parameterbereiche und Produktionskapazitäten. Zusätzlich umfasst die IST-Konfiguration die Positionierung der aktuell eingesetzten CPPM innerhalb eines Layouts.

Ergibt ein Abgleich, dass die SOLL-Produktion mit der IST-Konfiguration nicht durchführbar ist, erfolgt die Generierung alternativer Konfigurationen, die im Folgenden beschrieben wird, andernfalls wird auf einen weiteren Trigger gewartet.

(2) Generierung alternativer Konfigurationen: Sobald festgestellt wurde das Rekonfigurationsbedarf besteht, beginnt die Generierung alternativer Konfigurationen, deren Ablauf in Bild 3 visualisiert ist. Zunächst findet eine Generierung von Alternativen auf Maschinenebene statt. Das Ziel auf dieser Ebene ist neue Konfigurationsvorschläge für die verschiedenen CPPM zu finden, die zumindest Teile der vorgegebenen Produktionssequenz durchführen können. Diese Konfigurationsvorschläge umfassen sowohl die Soft- als auch die Hardware der jeweiligen CPPM. Daher basiert dieser Schritt auf den gegebenen Anforderungen sowie auf den Fähigkeiten (Operationen) der CPPM. 


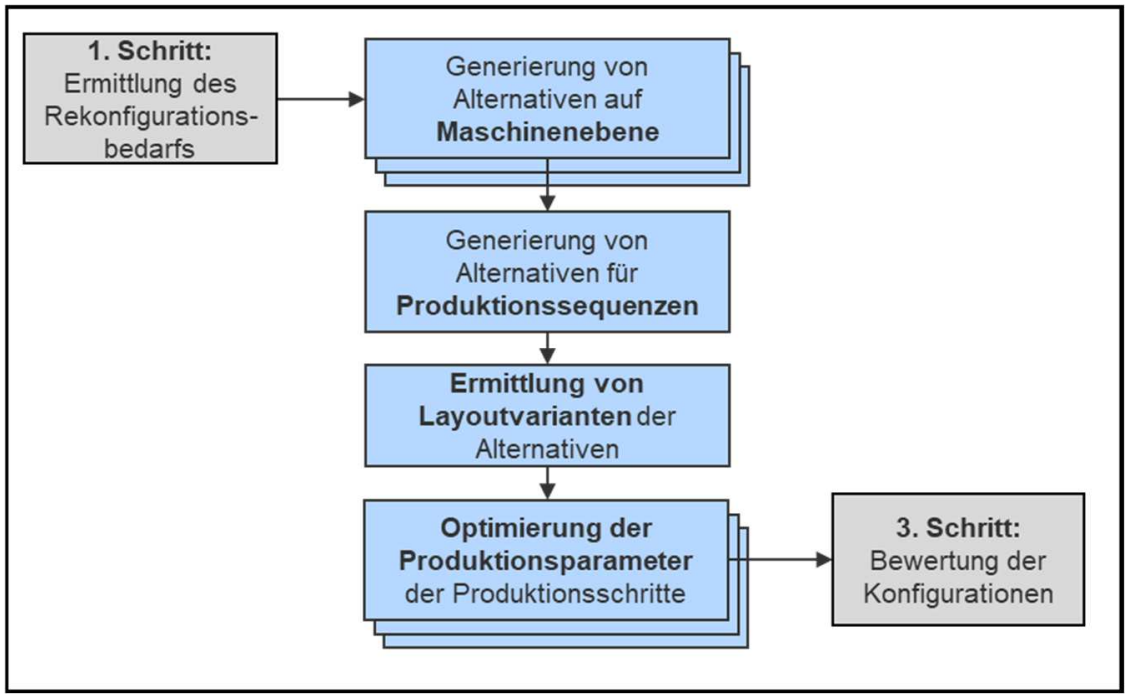

Bild 3: Generierung alternativer Konfigurationen (vereinfachte Darstellung)

Hierbei sollen die Fähigkeiten mit den, durch die Anforderungen geforderten, Produktionsprozessen verglichen werden können. Diese Fähigkeiten können mit ihren entsprechenden Software- und Hardware-Teilen verknüpft werden, um eine skalierbare Granularität zu erreichen. Neben den alternativen Konfigurationen der CPPM welche neue Fähigkeiten durch eine Rekonfiguration anbieten können, werden auch die aktuellen CPPMKonfigurationen mit den Fähigkeiten, die sie bereits besitzen berücksichtigt. Darüber hinaus können auch CPPM, die nicht Teil der momentanen IST-Konfiguration sind, auf dieser Ebene in Betracht gezogen werden.

Es wird also ein Lösungsraum aufgespannt, der aus denjenigen möglichen Konfigurationen aller CPPM besteht, welche mindestens einen Produktionsprozess der geforderten Produktionssequenz anbieten können.

Um die gesamte erforderliche Produktionssequenz abzudecken, wird anschließend die Generierung von Alternativen für Produktionssequenzen durchgeführt. Hier werden alle möglichen Varianten gebildet, die in der Lage sind, die gesamte geforderte Produktionssequenz umzusetzen. Diese Varianten bestehen jeweils aus den verschiedenen CPPM, wobei sich jedes in einer der möglichen Konfigurationen befindet, die sich in dem oben genannten Lösungsraum ergeben haben. Für jede Variante werden die einzelnen CPPM einem oder mehreren Produktionsprozessen zugeordnet.

Nach der Ermittlung der möglichen Alternativen für Produktionssequenzen müssen die CPPM durch die Ermittlung von Layoutvarianten der Alternativen im Layout des Produktionssystems positioniert werden. Grundlage hierfür ist die Beschreibung der vorgegebenen Layout-Struktur. Bei der Bildung dieser verschiedenen Varianten ist es entscheidend, den Aufwand für diese Rekonfigurationsmaßnahmen auf Systemebene, 
ausgehend von der derzeit angewandten Konfiguration des CPPS, zu bestimmen. Dieser Aufwand wird im Hinblick auf die ausgewählten Kriterien (Zeit, Kosten und Energie) berechnet, um später einen Vergleich der Varianten zu ermöglichen.

Im letzten Teilschritt der Generierung alternativer Konfigurationen wird die Optimierung der Produktionsparameter der Produktionsschritte durchgeführt. Diese Optimierung zielt auf das beste Ergebnis in Bezug auf die gegebenen gewichteten Kriterien (Zeit, Kosten und Energie) ab. Daher basiert sie auf der Beschreibung der Konditionen zu denen die CPPM ihre Fähigkeiten einsetzen können. Das Ergebnis dieses Teilschritts ist ein Satz von optimierten Produktionsparametern für jede Variante, sowie das eigentliche Optimierungsergebnis, das später für einen Vergleich herangezogen werden kann. Folglich sind nun alle möglichen Konfigurationen des CPPS gefunden worden und können bewertet und miteinander verglichen werden.

(3) Bewertung der Konfigurationen: Für die Bewertung werden für alle zuvor gefundenen Konfigurationen die Optimierungsergebnisse herangezogen, welche die Gewichtung der Kriterien berücksichtigen. Darüber hinaus wird der Aufwand für die Durchführung der Rekonfigurationsmaßnahmen entsprechend des oben beschriebenen Schritts der Generierung von Alternativen berücksichtigt.

(4) Auswahl einer Konfiguration: Anschließend findet die Auswahl einer neuen Konfiguration statt. Um die am besten geeignete Konfiguration zu bestimmen, werden alle Varianten anhand ihrer in Schritt 3 ermittelten Bewertung verglichen. Das Gesamtergebnis dieser Methodik ist die neue Konfiguration für das CPPS. Diese umfasst sowohl die Konfiguration auf Maschinen(jeweilige CPPM) als auch auf Systemebene (gesamtes CPPS), einschließlich der Positionierung der einzelnen CPPM innerhalb des Layouts des CPPS, sowie die ermittelten Produktionsparameter. Diese optimierten Produktionsparameter können dann von der Produktionsverwaltung verwendet werden.

\subsection{Zuordnung der Intelligenz}

Die Intelligenz für das Rekonfigurationsmanagement, welche die in Abschnitt 3.1 beschriebene Methodik umsetzt, kann im CPPS entsprechend der in Abschnitt 2.2 eingeführten Struktur verortet werden. Die vorgeschlagene Verortung folgt der Idee, einen dynamischen Ein- und Austritt von Produktionsressourcen und den Umgang mit ihrer Heterogenität zu erleichtern und ist in Bild 4 dargestellt. Die Intelligenz der CPPM kann jeweils auf unterschiedliche Weise mit dessen Modellen interagieren, dies kann z.B. von den Eigenschaften der Hardware oder den Herstellerrichtlinien und dem daraus resultierenden Modell abhängen. Allerdings sind standardisierte Schnittstellen zu der Intelligenz, die dem gesamten CPPS zugeordnet ist, erforderlich. 


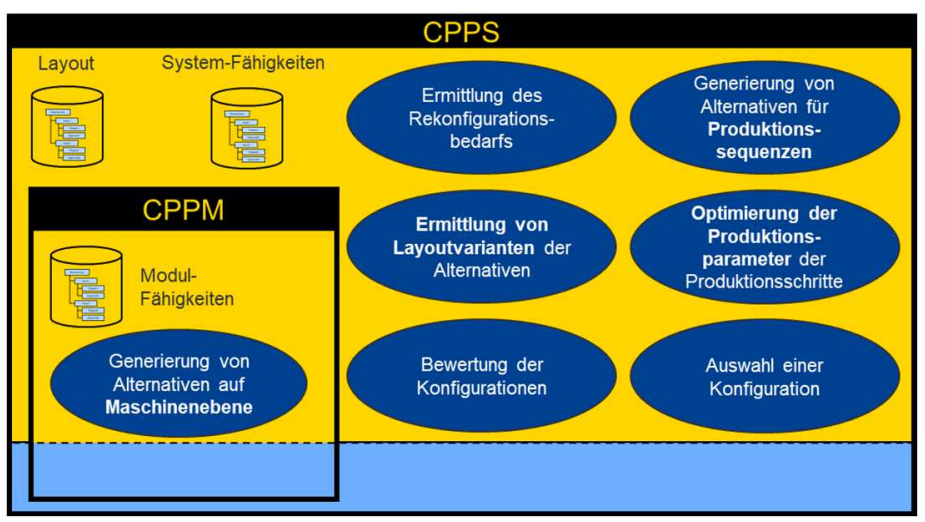

Bild 4: Verortung der Intelligenz innerhalb des CPPS

Darüber hinaus ermöglicht diese Zuordnung, dass Konfigurationen auf Maschinenebene bei Bedarf auf unterschiedliche Weisen generiert werden können.

\section{Realisierung}

Zunächst wurde, wie in Bild 5 (links) dargestellt, ein modulares Produktionssystem mit MatrixLayout, simuliert in Unity und gesteuert über ein verteiltes serviceorientiertes OPC-UASteuerungsnetzwerk, prototypisch aufgebaut. Dieses wurde von dem in [29] vorgestellten physischen modularen Produktionssystem und seiner Steuerung inspiriert. Dies wird durch die Drag \& Drop-Funktionalität innerhalb der Unity-Simulation, gepaart mit der Plug \& ProduceFähigkeit des serviceorientierten Steuerungsnetzwerks ermöglicht. Des Weiteren zeigt Bild 5 (rechts) zeigt das reale CPPS, welches zukünftig als Demonstrator eingesetzt wird.
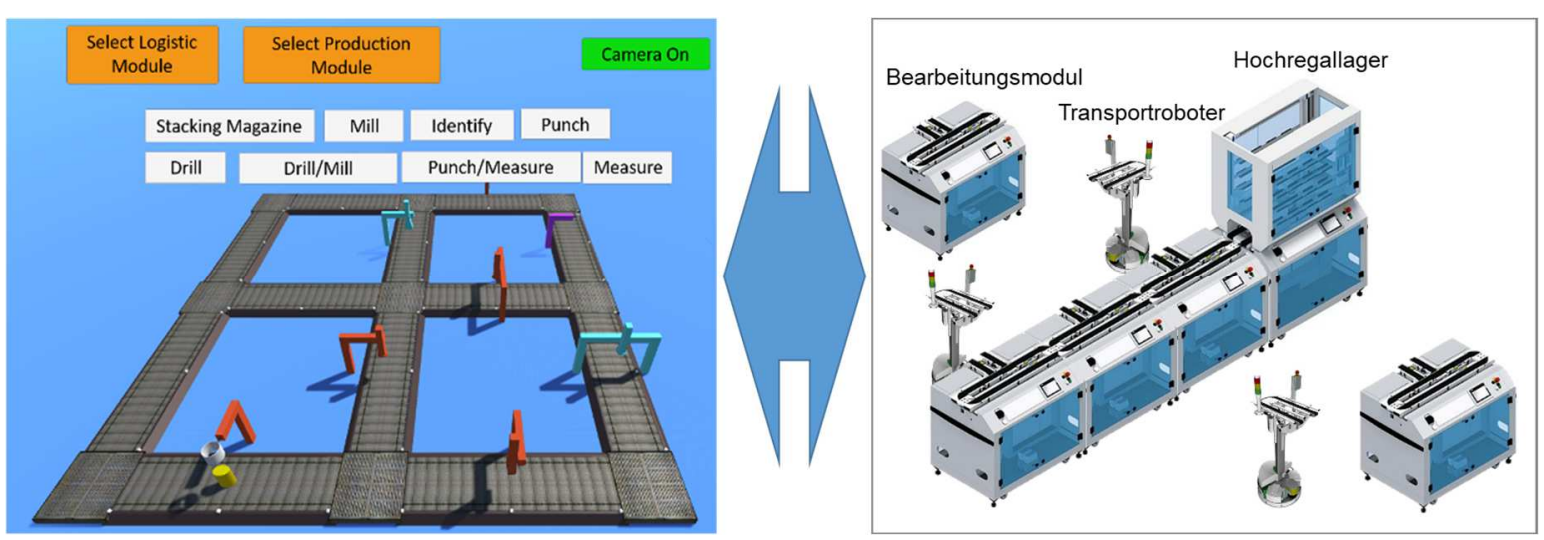

Bild 5: Simuliertes modulares Produktionssystem (links); Reales Cyber-Physisches Produktionssystem (in Beschaffung befindlich) (rechts)

Die Intelligenz für das Rekonfigurationsmanagement wurde in vereinfachter Form durch ein Agentensystem realisiert. Das implementierte Rekonfigurationsmanagement basiert auf einem zweistufigen genetischen Algorithmus zur Generierung von Konfigurationen und einem $A^{*}$ Algorithmus zur Bestimmung des Materialflusses. Das Ergebnis ist eine neue Konfiguration, die in der Lage ist, die erforderliche Produktionssequenz auszuführen. Die Konfiguration besteht aus den CPPM und deren Positionierung innerhalb des Layouts des CPPS. Dieser 
Prototyp ermöglicht eine einfache Validierung der aktuellen Ergebnisse, sowie der Ergebnisse nach der vollständigen Umsetzung des Konzepts.

\section{Zusammenfassung und Ausblick}

Der Beitrag stellt die Verbreitung von CPPS, sowie der zunehmende Bedarf von Rekonfigurationen während des Betriebs, in der zukünftigen industriellen Automatisierung dar. Das Thema der Rekonfiguration wird beleuchtet und aktuelle Schwachstellen werden identifiziert, derzeitige Rekonfigurationen sind demnach zeitaufwändig, fehleranfällig, nicht objektiv und liefern keine Garantie für eine optimumnahe Lösung. Aufbauend werden CPPS und ihre Potentiale zur Überwindung dieser Schwachstellen, insbesondere, dass diese über Modelle verfügen und Intelligenz anbieten können, diskutiert. Die sich ergebende Schlussfolgerung zeigt auf, dass die Nutzung dieser Potentiale ein neuartiges Konzept für ein selbstorganisiertes Rekonfigurationsmanagement erfordert. Daher wird ein neuartiges Konzept präsentiert, um die folgende Forschungsfrage zu beantworten: Wie können CPPS um die Fähigkeit eines selbstorganisierten Rekonfigurationsmanagements bereichert werden? Dies wird durch die

- Beschreibung der Methodik für das Rekonfigurationsmanagement und

- Verortung der Intelligenz, zur Umsetzung dieser Methodik, innerhalb des CPPS erreicht.

Der Artikel beschreibt die aktuelle Realisierung an einem simulierten modularen Produktionssystem und stellt ein reales CPPS für die zukünftige Umsetzung vor.

Zukünftig wird das in Beschaffung befindliche Cyber-Physische Produktionssystem für die weitere Realisierung und anschließende Evaluation eingesetzt. Hierzu wird die konkrete Methodik verfeinert, die für die Generierung von Alternativen auf Maschinenebene und für die Optimierung der Produktionsparameter eingesetzt wird. Dies beeinflusst auch die Modellierung des CPPS und seiner CPPM, welche spezifiziert werden muss. Dabei wird insbesondere das Konzept des digitalen Zwillings verwendet, mit dessen Hilfe Konfigurationsalternativen simulationsbasiert optimiert und getestet werden können.

\section{Literaturverzeichnis}

[1] N. Jazdi, "Cyber physical systems in the context of Industry 4.0," in 2014 IEEE international conference on automation, quality and testing, robotics, 2014, pp. 1-4.

[2] B. Bagheri, S. Yang, H.-A. Kao, and J. Lee, "Cyber-physical systems architecture for self-aware machines in industry 4.0 environment," IFAC-PapersOnLine, vol. 48, no. 3, pp. 1622-1627, 2015. 
[3] G. Lanza, P. Nyhuis, J. Fisel, A. Jacob, and L. Nielsen, "Wandlungsfähige menschzentrierte Strukturen in Fabriken und Netzwerken der Industrie 4.0 "(acatech Studie) München," Herbert Utz Verlage, 2018.

[4] E. Geisberger and M. Broy, "agendaCPS: Integrierte Forschungsagenda Cyber-Physical Systems," vol. 1, 2012.

[5] B. Vogel-Heuser et al., "Interdisciplinary engineering of cyber-physical production systems: highlighting the benefits of a combined interdisciplinary modelling approach on the basis of an industrial case," Design Science, vol. 6, 2020.

[6] E. Järvenpää, N. Siltala, and M. Lanz, "Formal resource and capability descriptions supporting rapid reconfiguration of assembly systems," in 2016 IEEE International Symposium on Assembly and Manufacturing (ISAM), 2016, pp. 120-125.

[7] C. Müller-Schloer, H. Schmeck, and T. Ungerer, "Organic Computing," Informatik Spektrum, vol. 35, no. 2, 2012, doi: 10.1007/s00287-012-0599-2.

[8] B. Vogel-Heuser, A. Fay, I. Schaefer, and M. Tichy, "Evolution of software in automated production systems: Challenges and research directions," Journal of Systems and Software, vol. 110, pp. 54-84, 2015.

[9] Y. Koren, X. Gu, and W. Guo, "Reconfigurable manufacturing systems: Principles, design, and future trends," Frontiers of Mechanical Engineering, vol. 13, no. 2, 2018.

[10] E. Järvenpää, N. Siltala, O. Hylli, and M. Lanz, "Capability matchmaking procedure to support rapid configuration and re-configuration of production systems," Procedia Manufacturing, vol. 11, pp. 1053-1060, 2017.

[11] A. M. A. Youssef and H. A. EIMaraghy, "Optimal configuration selection for reconfigurable manufacturing systems," International Journal of Flexible Manufacturing Systems, vol. 19, no. 2, pp. 67-106, 2007.

[12] J. Matevska, "Rekonfiguration komponentenbasierter Softwaresysteme zur Laufzeit,"Springer, 2010.

[13] T. Stehle and U. Heisel, "Konfiguration und Rekonfiguration von Produktionssystemen," in Neue Entwicklungen in der Unternehmensorganisation: Springer, 2017, pp. 333-367.

[14] F. Karl, "Bedarfsermittlung und Planung von Rekonfigurationen an Betriebsmitteln,"Herbert Utz Verlag, 2015.

[15] X. L. Hoang, A. Fay, P. Marks, and M. Weyrich, "Systematization approach for the adaptation of manufacturing machines," in 2016 IEEE 21st international conference on emerging technologies and factory automation (ETFA), 2016, pp. 1-4. 
[16] M. N. Hansson, E. Järvenpää, N. Siltala, and O. Madsen, "Modelling Capabilities for Functional Configuration of Part Feeding Equipment," Procedia Manufacturing, vol. 11, pp. 2051-2060, 2017.

[17] A. F. Hees, "System zur Produktionsplanung für rekonfigurierbare Produktionssysteme,"Herbert Utz Verlag, 2017.

[18] M. Broy, M. V. Cengarle, and E. Geisberger, "Cyber-physical systems: imminent challenges," in Monterey workshop, 2012, pp. 1-28.

[19] L. Monostori, "Cyber-physical production systems: Roots, expectations and R\&D challenges," Procedia CIRP, vol. 17, pp. 9-13, 2014.

[20] K. D. Bettenhausen and S. Kowalewski, "Cyber-physical systems: Chancen und Nutzen aus Sicht der Automation," VDI/VDE-Gesellschaft Mess-und Automatisierungstechnik, pp. 9-10, 2013.

[21] E. A. Lee, "Cyber physical systems: Design challenges," in 2008 11th IEEE International Symposium on Object and Component-Oriented Real-Time Distributed Computing (ISORC), 2008, pp. 363-369.

[22] B. Maschler and M. Weyrich, "Deep Transfer Learning at Runtime for Image Recognition in Industrial Automation Systems," 16th Technical Conference EKA (Magdeburg), 2020.

[23] B. Lindemann, C. Karadogan, N. Jazdi, M. Liewald, and M. Weyrich, "Cloud-based control approach in discrete manufacturing using a self-learning architecture," IFACPapersOnLine, vol. 51, no. 10, pp. 163-168, 2018.

[24] J. Schlick, P. Stephan, and T. Greiner, "Kontext, Dienste und Cloud Computing," atp magazin, vol. 55, no. 04, pp. 32-41, 2013.

[25] VDMA, "Industrie 4.0 - Research at German Research Institutes - an Overview," 2019.

[26] M. Ehrlich, L. Wisniewski, H. Trsek, and J. Jasperneite, "Modelling and automatic mapping of cyber security requirements for industrial applications: Survey, problem exposition, and research focus," in 2018 14th IEEE International Workshop on Factory Communication Systems (WFCS), 2018, pp. 1-9.

[27] S. Weyer, T. Meyer, M. Ohmer, D. Gorecky, and D. Zühlke, "Future modeling and simulation of CPS-based factories: an example from the automotive industry," IFACPapersOnLine, vol. 49, no. 31, pp. 97-102, 2016.

[28] B. Ashtari Talkhestani, D. Braun, W. Schloegl, and M. Weyrich, "Qualitative and quantitative evaluation of reconfiguring an automation system using Digital Twin," 2020.

[29] J.-P. Schmidt, T. Müller, and M. Weyrich, "Methodology for the model driven development of service oriented plant controls," Procedia CIRP, vol. 67, pp. 173-178, 2018. 